\title{
Deltonin induces apoptosis in MDA-MB-231 human breast cancer cells via reactive oxygen species-mediated mitochondrial dysfunction and ERK/AKT signaling pathways
}

\author{
SHIYUAN ZHANG ${ }^{1 *}$, YANG HE ${ }^{1 *}$, QINGYI TONG ${ }^{1}$, QIANMING CHEN ${ }^{2}$, XIAOHUA WU $^{1}$ and WEN HUANG ${ }^{1}$ \\ ${ }^{1}$ Laboratory of Ethnopharmacology, Regenerative Medicine Research Center, Institute for Nanobiomedical Technology and \\ Membrane Biology, West China Hospital/Medical School, Sichuan University, Sichuan 610041; \\ ${ }^{2}$ State Key Laboratory of Oral Disease, West China Hospital of Stomatology, \\ Sichuan University, Chengdu 610041, P.R. China
}

Received September 9, 2012; Accepted December 28, 2012

DOI: $10.3892 / \mathrm{mmr} .2013 .1273$

\begin{abstract}
Deltonin, a steroidal saponin isolated from Dioscorea zingiberensis Wright, exhibits high cytotoxic activity in cancer cells. In the present study, the effects of deltonin on cell proliferation and apoptosis were evaluated in the MDA-MB-231 human breast carcinoma cell line. Following treatment with deltonin, the viability of MDA-MB-231 cells was analyzed using MTT assay and apoptosis, mitochondrial membrane potential $(\Delta \Psi \mathrm{m})$ alternation and intracellular reactive oxygen species (ROS) generation was determined by flow cytometry. In addition, western blot analysis was performed to examine the expression of apoptosis-associated proteins. The results demonstrated that deltonin induced apoptosis in MDA-MB-231 cells in a time- and concentration-dependent manner. Apoptosis was associated with depolarization of $\Delta \Psi \mathrm{m}$ and time-dependent ROS generation. Deltonin treatment also resulted in Bax upregulation, Bcl-2 downregulation, activation of caspase- 3 and -8 and poly (ADP ribose) polymerase cleavage. Decreased levels of phosphorylated extracellular signal-regulated kinase (ERK) and phosphorylated AKT were also observed. Results indicate that the proliferation inhibi-
\end{abstract}

Correspondence to: Professor Wen Huang or Dr Xiaohua Wu, Laboratory of Ethnopharmacology, Regenerative Medicine Research Center, Institute for Nanobiomedical Technology and Membrane Biology, West China Hospital/Medical School, Sichuan University, 1 Keyuan 4 Road, No. 1 Gaopeng Avenue, Sichuan 610041, P.R. China E-mail: huangwen@scu.edu.cn

E-mail:wuxh@scu.edu.cn

Professor Qianming Chen, State Key Laboratory of Oral Disease, West China Hospital of Stomatology, Sichuan University, Chengdu 610041, P.R. China

E-mail: qmchen@scu.edu.cn

*Contributed equally

Key words: breast carcinoma, deltonin, apoptosis, reactive oxygen species, ERK/AKT tory effect of deltonin is associated with its apoptosis-inducing effect, which may correlate with ROS-mediated mitochondrial dysfunction as well as activation of the ERK/AKT signaling pathways. Therefore, deltonin may be a potential chemotherapeutic agent for the treatment of breast cancer.

\section{Introduction}

Breast cancer is the most commonly diagnosed cancer and the leading cause of cancer mortality among females, accounting for $23 \%$ of total cancer cases and $14 \%$ of cancer mortalities (1). In developing countries, including China, breast cancer remains a significant public health issue due to its prevalence (2). In recent years, medical advances have increased the availablity and efficacy of breast cancer treatments, including improved surgical methods for lumpectomy and mastectomy and radiation, hormone and chemotherapies. However, the majority of drugs currently used as chemotherapeutic agents exhibit low efficacy and are associated with the development of drug resistance $(3,4)$. Therefore, new drugs with a higher therapeutic index are urgently required to effectively treat this malignancy.

Over the last two decades, an increasing number of bioactive compounds have been identified in traditional Chinese medical herbs (5). Specific compounds have been reported to kill tumor cells by generation of reactive oxygen species (ROS). ROS are known to affect mitochondrial membrane potential $\left(\Delta \Psi_{\mathrm{m}}\right)$ and trigger a series of mitochondria-associated events $(6,7)$. The generation of ROS may contribute to mitochondrial damage, reduction of $\Delta \Psi \mathrm{m}$, release of cytochrome $\mathrm{c}$ and Smac and subsequent caspase activation and apoptosis $(8,9)$. Apoptosis is a major control mechanism of cell death when DNA damage is not repaired. Apoptosis is a gene-directed programmed cell death characterized by cell shrinkage, blebbing of the plasma membrane, chromosomal DNA fragmentation and a number of other morphological alterations (10). There are two major pathways of apoptosis, the cell death receptor- (extrinsic) and mitochondria-mediated apoptotic (intrinsic) pathways (11). Mitochondria are central to the intrinsic apoptotic pathway. Disruption of $\Delta \Psi \mathrm{m}$, loss of mitochondrial potential and induction of cytochrome $\mathrm{c}$ release from the mitochondria into the cytosol 
are all associated with regulation of apoptosis and are recognized as key steps in the mitochondrion-dependent apoptotic pathway (12-14). Deltonin, diosgenin-3-O- $\beta$-D-glucopyranosyl $(1 \rightarrow 4)$-[ $\alpha$-L-rhamnopyranosyl $(1 \rightarrow 2)]-\beta$-D-glucopyranoside, is a steroidal saponin. The antiproliferative and apoptosis-inducing effects of steroidal saponins, including compounds isolated from plants and synthesized analogs, have been demonstrated in vitro using a number of cancer cell lines (15-18). We have previously demonstrated that deltonin has cytotoxic effects against colon cancer cell lines and oral administration of deltonin was found to significantly inhibit tumor growth and prolonged survival of tumor bearing mice $(19,20)$. However, the effects of deltonin in breast cancer remain unknown. To elucidate the anticancer activity and mechanisms by which deltonin functions, the effects of deltonin on cell proliferation and apoptosis in the MDA-MB-231 human breast carcinoma cell line were evaluated. Deltonin induced apoptosis through loss of $\Delta \Psi \mathrm{m}$, accumulation of intracellular ROS, caspase activation, regulation of Bcl-2 family members and modulation of extracellular signal-regulated kinase (ERK)/AKT signaling pathways.

\section{Materials and methods}

Reagents. Deltonin was obtained as previously described and its purity determined by high performance liquid chromatography (>98\%) (19). The compound was prepared as a stock solution in DMSO [final concentration $<0.05 \%$ $(\mathrm{v} / \mathrm{v})]$ and diluted in the relevant culture medium. ROS assay and JC-1 $\Delta \Psi \mathrm{m}$ detection kits were obtained from Beyotime Institute of Biotechnology (Jiangsu, China). 3-(4,5-Dimethylthiazol-2-yl)-2,5-diphenyltetrazolium (MTT), DMSO, N-acetylcysteine (NAC) and primary antibody against GAPDH were purchased from Sigma-Aldrich (St. Louis, MO, USA). Primary antibodies against caspase-3 and -8, ERK1/2, phospho-ERK1/2 (Thr202/204), AKT and phospho-AKT (Ser473) were purchased from Cell Signaling Technology (Beverly, MA, USA). Primary antibodies against Bcl-2 (C-2), Bax (P19) and secondary antibodies were purchased from Santa Cruz Biotechnology, Inc. (Santa Cruz, CA, USA). All other chemicals and reagents were of the highest purity grade commercially available.

Cell lines and cell culture. Human breast carcinoma cell line, MDA-MB-231, was obtained from the American Type Culture Collection (Manassas, VA, USA) and maintained in RPMI-1640 medium containing $10 \%$ fetal bovine serum (Gibco-BRL, Auckland, New Zealand), $100 \mathrm{U} / \mathrm{ml}$ penicillin and $100 \mu \mathrm{g} / \mathrm{ml}$ streptomycin in a humid chamber at $37^{\circ} \mathrm{C}$ under $5 \% \mathrm{CO}_{2}(21)$.

Cytotoxicity assay. The inhibitory effect of deltonin on cell viability was measured by MTT assay (22). Cells were seeded in 96-well plates (Costar Corning, Rochester, NY, USA) at a density of $3 \times 10^{3}$ cells/well. Deltonin underwent serial dilution $(0.5,1.0,2.0,4.0,8.0 \mu \mathrm{M})$ and DMSO $(<0.05 \%)$ was used as a control. Absorbance at $570 \mathrm{~nm}$ was measured with Spectra Max M5 (Molecular Devices, LLC, Sunnyvale, CA, USA) following drug treatment $(12,24,36,48 \mathrm{~h})$. All experiments were performed in triplicate. Dose- and time-dependent curves of deltonin-treated MDA-MB-231 cells were generated as the percentage cell growth inhibition, using the following formula: $\%$ inhibition $=1-\mathrm{A} 570_{\text {treated cells }} / \mathrm{A} 570_{\text {control cells }} \mathrm{x} 100$. The $50 \%$ inhibiting concentration was calculated using SPSS software v13.0.

Flow cytometry assay for apoptosis. The use of Annexin V-fluorescein isothiocyanate (FITC) and propidium iodide (PI) for flow cytometry provides a rapid and convenient assay for apoptosis as described previously (23). Cells were exposed to desired concentrations of deltonin for $24 \mathrm{~h}$ and the apoptotic index was assessed using an Annexin V-FITC apoptosis kit (KeyGEN, Nanjing, China) according to the manufacturer's instructions. For each analysis, 30,000 events were acquired on a forward and side scatter gate. Annexin V-positive PI-negative cells represented early apoptotic populations and Annexin V-positive PI-positive cells represented late apoptotic or secondary necrotic populations. Data acquisition and analysis were performed by fluorescence-activated cell sorting using list mode software (Becton-Dickinson, Franklin Lakes, NJ, USA).

Measurement of $\Delta \Psi_{m}$. Alterations in $\Delta \Psi \mathrm{m}$ were analyzed by flow cytometry using a $\Delta \Psi \mathrm{m}$-sensitive molecular probe dye, JC-1, as described previously (24). The JC-1 dye bearing a delocalized positive charge enters the mitochondrial matrix due to the negative charge established by the intact $\Delta \Psi \mathrm{m}$. In healthy cells, JC-1 dye stains the mitochondria red due to formation of JC-1 aggregates. In apoptotic cells, JC-1 dye accumulates in the cytoplasm in monomeric form (green fluorescence) due to collapse of $\Delta \Psi \mathrm{m}$. Cells were treated with $3.0 \mu \mathrm{M}$ deltonin for $0,4,8,12,16$ and 24 h. Following this, cells were harvested, washed once in cold PBS and incubated with JC-1 dye at $37^{\circ} \mathrm{C}$ for $20 \mathrm{~min}$. Stained cells were washed and re-suspended in $0.5 \mathrm{ml}$ assay buffer and the fluorescence was measured using flow cytometry. The emission wavelengths of JC-1 monomers and JC-1 aggregates were 530 and $590 \mathrm{~nm}$, respectively.

Measurement of intracellular ROS. Intracellular ROS generation was monitored using an ROS assay kit and flow cytometry using fluorescence produced by $2^{\prime}, 7^{\prime}$-dichlorofluorescein following oxidation from 2',7'-dichlorfluorescein-diacetate (DCFH-DA; Molecular Probes, Grand Island, NY, USA) (25). In brief, following treatment with $3.0 \mu \mathrm{M}$ deltonin for $0,4,8$, 12,16 and $24 \mathrm{~h}$, cells were harvested and then incubated with $10 \mu \mathrm{M}$ DCFH-DA in a culture medium at $37^{\circ} \mathrm{C}$ for $30 \mathrm{~min}$. Cells were washed and re-suspended in PBS. ROS generation was measured by flow cytometry.

Inhibitor treatment. To confirm the role of intracellular ROS in deltonin-induced apoptosis, a common quencher of ROS, $\mathrm{NAC}$, was used to inhibit intracellular alteration of redox states (26). NAC was dissolved in PBS and adjusted to $\mathrm{pH} 7.4$ to produce a $0.1 \mathrm{M}$ stock solution. Cells were pre-incubated with $6 \mathrm{mM}$ NAC for $2 \mathrm{~h}$ and then treated with $3 \mu \mathrm{M}$ deltonin. After $8 \mathrm{~h}$, alterations in ROS production were determined by flow cytometry. After $24 \mathrm{~h}$, percentage of apoptotic cells and alterations in $\Delta \Psi \mathrm{m}$ were analyzed by flow cytometry.

Protein extraction and western blot analysis. Western blot analysis was performed as described previously (27). Cells 

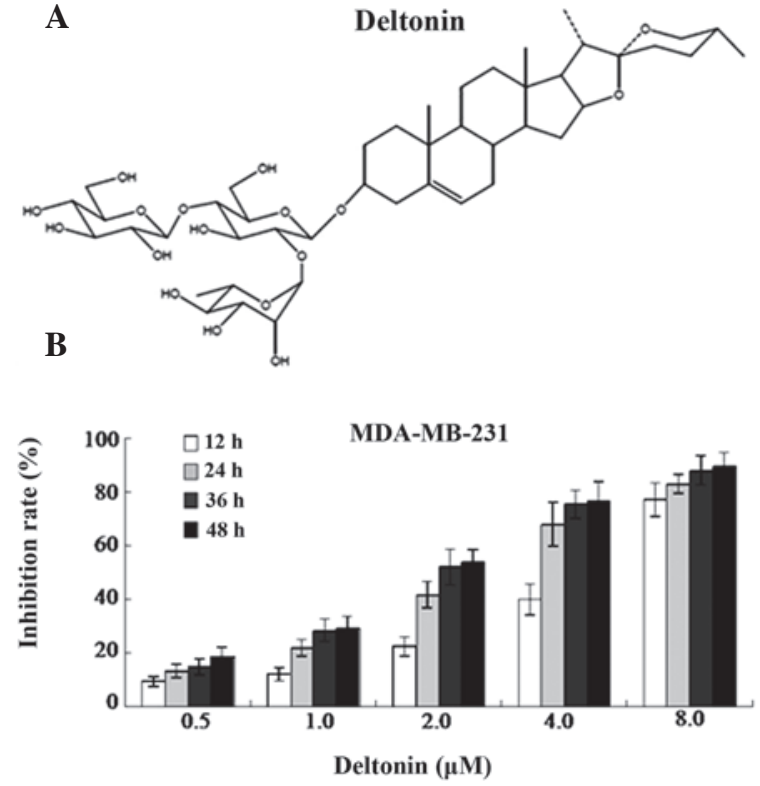

Figure 1. Chemical structure of deltonin and cytotoxic effects on MDA-MB-231 cells. (A) Chemical structure of deltonin. (B) MDA-MB-231 cells were treated with various concentrations of deltonin for 12, 24, 36 and $48 \mathrm{~h}$. Inhibition rate of deltonin was detected by MTT assay. Deltonin inhibited proliferation of MDA-MB-231 cells in a time- and dose-dependent manner. Values are presented as mean $\pm \mathrm{SE}$ of three experiments. $\mathrm{P}<0.05$, vs. control group. MTT, 3-(4,5-dimethylthiazol-2-yl)-2,5-diphenyltetrazolium.

(1x10\% $/$ well) were grown in 6-well microplates overnight and treated with various concentrations $(0,1,3,5 \mu \mathrm{M})$ of deltonin for $24 \mathrm{~h}$. Following this, cells were washed with ice-cold PBS and total cell lysates were prepared by RIPA buffer. Lysates was centrifuged at $12,000 \mathrm{~g}$ for $15 \mathrm{~min}$ at $4^{\circ} \mathrm{C}$. The supernatant was collected and total protein concentrations were determined using the BCA assay, dissolved in 5X SDS sample buffer and denatured. Proteins were separated on 10-15\% SDS-PAGE and transferred to polyvinylidene fluoride membrane (Bio-Rad, Hercules, CA, USA). Membranes were incubated overnight at $4^{\circ} \mathrm{C}$ with the respective primary antibodies [caspase- 3 and -8 , poly (ADP ribose) polymerase (PARP), Bcl-2, Bax, ERK1/2, phospo-ERK1/2, AKT and phospo-AKT] and horseradish peroxidase-conjugated secondary antibodies at $37^{\circ} \mathrm{C}$ for $1 \mathrm{~h}$. Reactive bands were identified using an enhanced chemiluminescent substrate to horseradish peroxidase (Amersham Pharmacia Biotech, Amersham, UK). Expression of GAPDH was used as a control.

Statistical analysis. Data are presented as mean \pm SE and were analyzed for statistical significance using analysis of variance, followed by Scheffe's test for multiple comparisons. $\mathrm{P}<0.05$ was considered to indicate a statistically significant difference.

\section{Results}

Deltonin decreases cell viability and induces apoptosis. The chemical structure of deltonin is presented in Fig. 1A. The cytotoxic effects of deltonin were investigated by MTT assay. As demonstrated in Fig. 1B, the maximum inhibition ratio obtained with $8 \mu \mathrm{M}$ deltonin treatment was $89.40 \%$. Deltonin
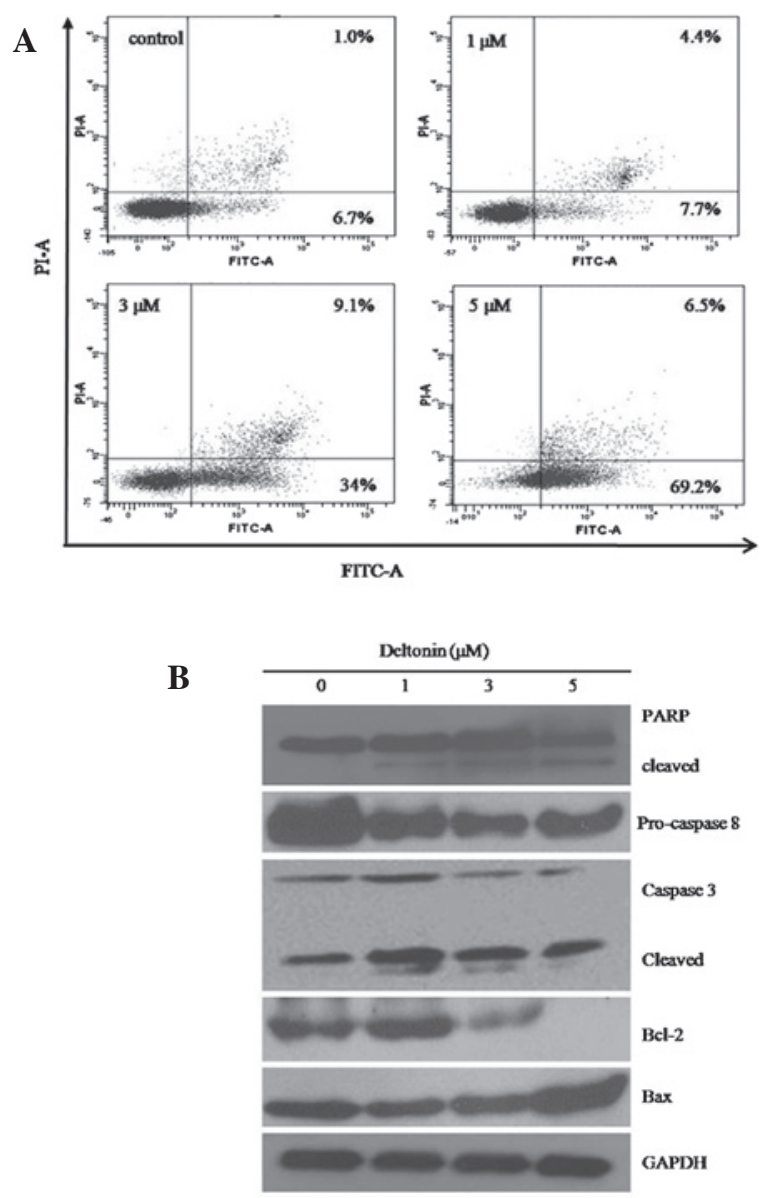

Figure 2. Deltonin induced apoptosis in MDA-MB-231 cells. (A) Flow cytometry analysis of Annexin V-FITC and PI staining to determine apoptosis in $24 \mathrm{~h}$ deltonin-treated MDA-MB-231 cells. (B) Western blot analysis of caspase- 8 and $-3, \mathrm{Bcl}-2$, Bax and PARP protein expression. GAPDH was used as a loading control. FITC, fluorescein isothiocyanate; PI, propidium iodide; PARP, poly (ADP ribose) polymerase.

inhibited the proliferation of MDA-MB-231 cells in a timeand concentration-dependent manner.

Flow cytometry analysis with an Annexin V-FITC apoptosis kit was also performed to identify the effects of deltonin. As shown in Fig. 2A, deltonin treatment induced apoptosis in MDA-MB-231 cells in a concentration-dependent manner. Compared with untreated cells, cells treated with $5 \mu \mathrm{M}$ deltonin resulted in up to $75.7 \%$ apoptosis incidence, indicating that deltonin causes death in MDA-MB-231 cells by the induction of apoptosis.

Effect of deltonin on the expression of apoptosis-related proteins. Apoptosis is executed by the coordinated actions of caspase family members and is tightly regulated by $\mathrm{Bcl}-2$ protein family members. Therefore, protein expression levels of these molecules were analyzed by western blot analysis. As demonstrated in Fig. 2B, treatment of MDA-MB-231 cells with deltonin led to activation of caspase-3 and -8. It is well known that activation of caspase-3 during apoptosis causes the cleavage of PARP, a major apoptotic enzyme. Deltonin increased levels of cleaved PARP in a dose-dependent manner, consistent with deltonin-induced apoptosis in MDA-MB-231 cells. Following this, the protein expression levels of Bcl-2 


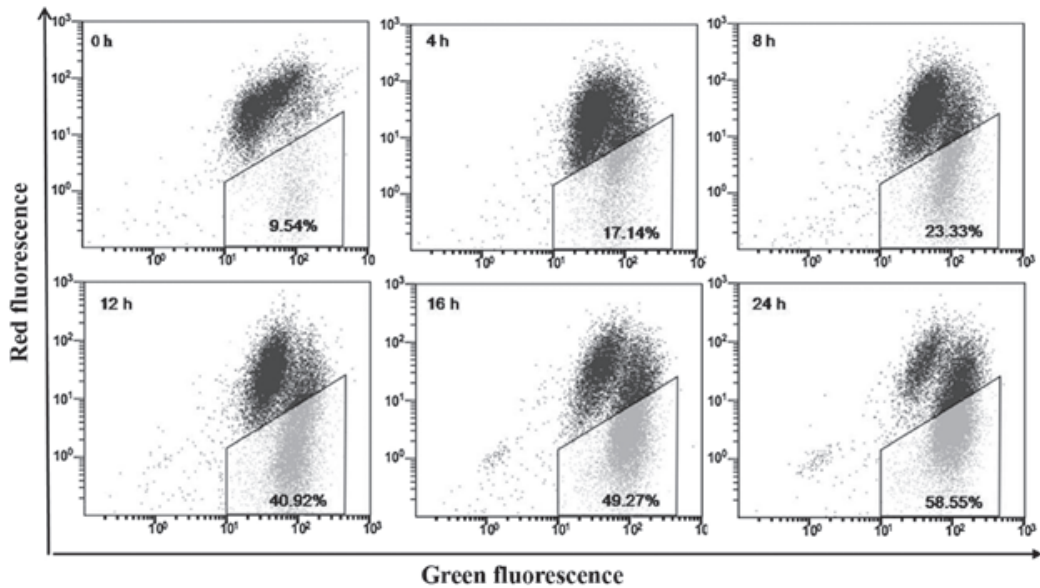

Figure 3. Deltonin induced dissipation of $\Delta \Psi \mathrm{m}$. MDA-MB-231 cells were treated with $3 \mu \mathrm{M}$ deltonin for $0,4,8,12,16$ and $24 \mathrm{~h}$, stained with JC-1 dye and analyzed by flow cytometry. Data are representative of three parallel experiments. $\Delta \Psi \mathrm{m}$, mitochondrial membrane potential.

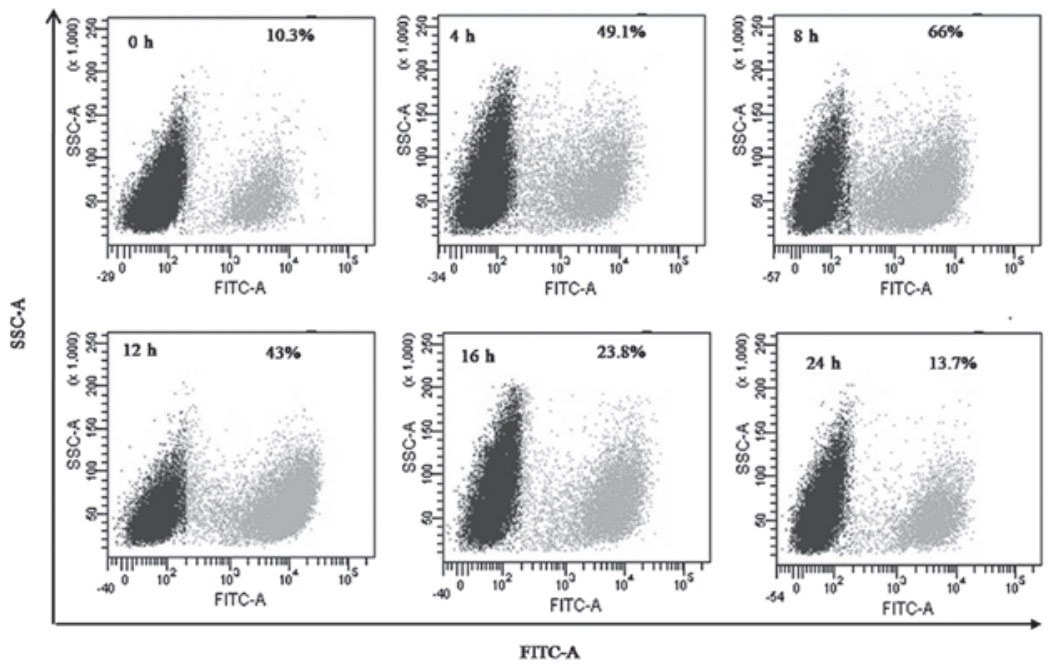

Figure 4. Deltonin induced generation of ROS. MDA-MB-231 cells were treated with $3 \mu \mathrm{M}$ deltonin for $0,4,8,12,16$ and $24 \mathrm{~h}$ and analyzed by flow cytometry. A marked increase in ROS levels was observed following $8 \mathrm{~h}$ of treatment and a decrease was observed at $24 \mathrm{~h}$ of treatment. Deltonin induced generation of ROS in a time-dependent manner. Data are representative of three parallel experiments. ROS, reactive oxygen species; FITC, fluorescein isothiocyanate.

family members, Bcl-2 and Bax were determined. Fig. 2B shows a decrease in Bcl-2 and increase in Bax levels.

Effect of deltonin on $\Delta \Psi m$ change. Change of $\Delta \Psi \mathrm{m}$ using JC-1 was performed to investigate whether mitochondria are involved in deltonin-induced apoptosis. Representative flow cytometric results are presented in Fig. 3. Deltonin treatment led to a time-dependent increase in the number of green fluorescence-positive cells from 9.5 in untreated cells to 17.1, $23.3,40.9,49.3$ and $58.6 \%$.

Effect of deltonin on intracellular ROS. $\triangle \Psi \mathrm{m}$ loss is an early event in apoptosis induction, which is often accompanied by ROS production. Intracellular ROS was examined using DCFH-DA to analyze the role of ROS in deltonin-induced apoptosis. As demonstrated in Fig. 4, treatment of MDA-MB-231 cells with $3 \mu \mathrm{M}$ deltonin for $0,4,8,12,16$ and $24 \mathrm{~h}$ resulted in ROS increase from 10.3 to $66 \%$ from 0 to $8 \mathrm{~h}$, however, ROS levels decreased to $13.7 \%$ at $24 \mathrm{~h}$. These results revealed that the ROS burst is generated in a time-dependent manner.
NAC inhibits deltonin-induced apoptosis. To determine the association between intracellular ROS and deltonin-induced apoptosis, MDA-MB-231 cells were pretreated with $6 \mathrm{mM}$ NAC for $2 \mathrm{~h}$ and then incubated with $3 \mu \mathrm{M}$ deltonin. As revealed in Fig. 5A, pretreatment with NAC reduced the number of apoptotic cells, whereby the percentage of apoptotic cells decreased from 32.9 to $23.4 \%$.

NAC inhibits deltonin-induced intracellular ROS accumulation. NAC markedly inhibited deltonin-induced accumulation of ROS, the intensity of DCFH-DA fluorescence decreased from 54.8 to $18.0 \%$ (Fig. $5 \mathrm{~B}$ ).

$N A C$ inhibits deltonin-induced depolarization of $\Delta \Psi m$. NAC is known to exert protective effects on cells, therefore, the ability of NAC to prevent depolarization of $\Delta \Psi \mathrm{m}$ in deltonin-treated cells was invetigated. Fig. 5C reveals flow cytometry analysis of $\Delta \Psi \mathrm{m}$ in the presence of absence of NAC $(6 \mathrm{mM})$ pretreament. The results demonstrate that deltonin-induced apoptosis in MDA-MB-231 cells may be 


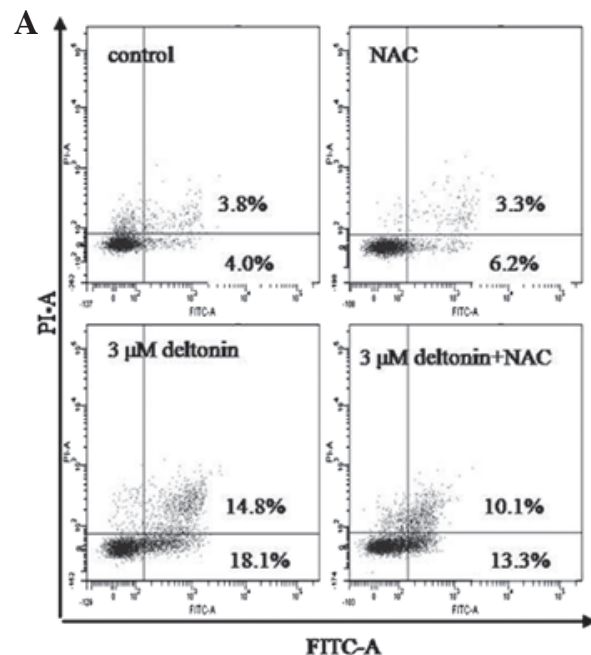

B
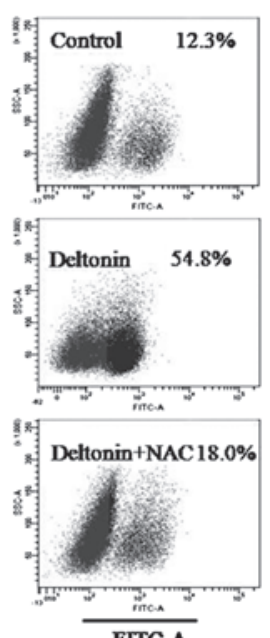

C
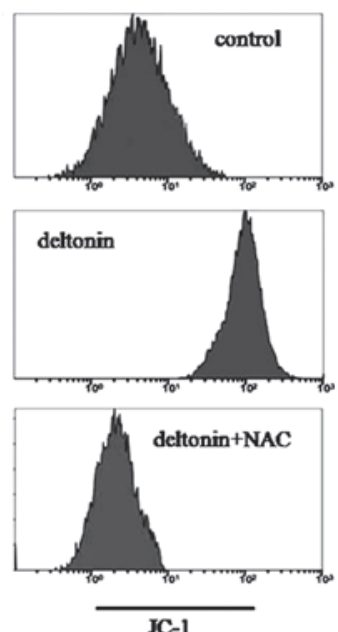

Figure 5. NAC antagonized deltonin-induced apoptosis in MDA-MB-231 cells. Cells were pretreated with 6 mM NAC for 2 h. (A) Effect of NAC on deltonin-induced apoptosis. Pretreated cells were treated with $3 \mu \mathrm{M}$ deltonin for $24 \mathrm{~h}$. (B) Effect of NAC on deltonin-induced ROS generation. Pretreated cells were treated with $3 \mu \mathrm{M}$ deltonin for $8 \mathrm{~h}$. (C) Effect of NAC on deltonin induced dissipation of $\Delta \Psi \mathrm{m}$. Pretreated cells were treated with $3 \mu \mathrm{M}$ deltonin for $24 \mathrm{~h}$. Data are representative of three parallel experiments. NAC, N-acetylcysteine; ROS, reactive oxygen species; FITC, fluorescein isothiocyanate; $\Delta \Psi \mathrm{m}$, mitochondrial membrane potential.

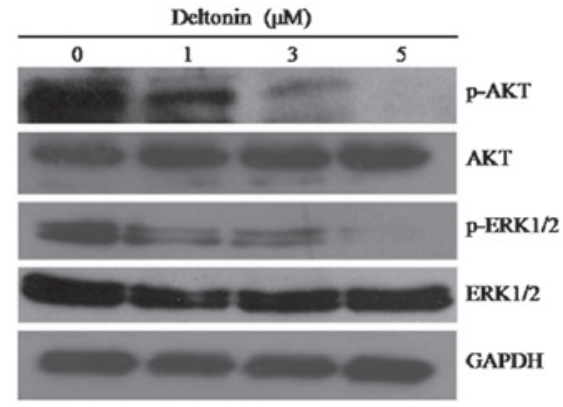

Figure 6. Deltonin inhibited phosphorylation of AKT and ERK1/2 in MDA-MB-231 cells. Total ERK1/2 and AKT slightly decreased. GAPDH was used as a loading control. ERK, extracellular signal-regulated kinase.

mediated by the mitochondrial pathway and, at least in part, by accumulation of ROS.

Effect of deltonin on the activation of ERKI/2 and AKT. ERK and AKT are two notable pathways associated with tumor development. Inhibition of the ERK pathway or AKT activation has been identified to induce apoptosis in tumor cells (28-30). To investigate the involvement of ERK/AKT signal pathways in deltonin-induced apoptosis of MDA-MB-231 cells, levels of phosphorylated and unphosphorylated AKT and ERK1/2 were determined by western blot analysis. As shown in Fig. 6 , treatment of deltonin decreased levels of phospo-ERK1/2 and -AKT in a dose-dependent manner compared with GAPDH (loading control). Results indicate that interference with ERK and AKT signal pathways may contribute to the anticancer activity of deltonin.

\section{Discussion}

Dioscorea zingiberensis Wright, a widely distributed medicinal plant, has been used extensively for a number of years in China. Deltonin is a major component of steroidal saponins from Dioscorea zingiberensis Wright and it has been reported to exhibit anticancer properties which may prove suitable for cancer therapy. In the present study, the effect of deltonin on breast cancer cells was evaluated and deltonin was found to significantly inhibit the growth of human breast cancer MDA-MB-231 cells in a time- and dose-dependent manner.

Apoptosis is a form of programmed cell death which occurs in response to a variety of stimuli and is characterized by a series of morphological and biochemical changes. Apoptosis is divided into two signaling pathways, death receptor-mediated (extrinsic) and mitochondrial (intrinsic) (31). Caspases are central to the majority of apoptotic responses. Activation of caspase- 3 is considered to initiate apoptosis and requires activation of initiator caspases, including caspase- 8 or -9 , in response to proapoptotic signals $(32,33)$. In the apoptotic assay, results of flow cytometry analysis confirmed that the percentage of apoptotic cells increased with deltonin concentration. In addition, the induction of apoptosis was confirmed by western blot analysis. Treatment of MDA-MB-231 cells with deltonin led to the degradation of caspase- 3 and -8 and cleavage of PARP in a dose-responsive manner. Bcl-2 family members are recognized as important regulators of apoptosis. In the current study, the expression levels of key Bcl-2 family proteins were determined. Western blot analysis demonstrated that deltonin downregulated $\mathrm{Bcl}-2$ levels and increased levels of Bax. Bcl-2 is an important regulator of apoptosis by control of mitochondrial function. Bcl-2 affects the release of cytochrome $\mathrm{c}$ from the mitochondria and activates a number of caspases $(34,35)$. Therefore, additional analysis focused on the mitochondrial pathway was performed.

Mitochondrial changes, including permeability transition pore opening and the collapse of $\Delta \Psi \mathrm{m}$, induces apoptosis by activation of caspases (36). In the present study, the treatment of MDA-MB-231 cells with deltonin resulted in the loss of $\Delta \Psi \mathrm{m}$ in a time-dependent manner. The results indicate that deltonin induced apoptosis of MDA-MB-231 cells, at least in part, through the mitochondrial damage-mediated caspase pathway. 
The role of ROS in mediating apoptosis in various cancer cells is well established (37). ROS generation causes functional disorder of mitochondria and leads to cell apoptosis $(38,39)$. Results indicate that deltonin induced generation of ROS and collapse of $\Delta \Psi \mathrm{m}$. Intracellular ROS levels were observed to significantly increase in a time-dependent manner and peaked at $8 \mathrm{~h}$ of deltonin treatment, indicating that deltonin may result in accumulation of intracellular ROS. To determine whether increased production of ROS was critical for deltonin-induced apoptosis, cells were pretreated with the ROS scavenger, NAC. Results revealed that NAC had a suppressive effect on deltonin-induced apoptosis, intracellular ROS generation and depolarization of $\Delta \Psi \mathrm{m}$, indicating that ROS is associated with activation of the mitochondrial pathway. In general, results demonstrate that deltonin induces apoptosis by a mechanism involving increased intracellular ROS levels in MDA-MB-231 cells.

AKT signaling is crucial for initiation and progression of breast cancer (40) and also regulates several downstream targets responsible for cell survival and proliferation (41-44). In the current study, deltonin downregulated expression of phospo-AKT, indicating that deltonin inhibits AKT-mediated survival signaling in breast cancer cells. In addition, the ERK signaling pathway is an important pathway associated with mediating cell growth, survival and death $(45,46)$. In the present study, deltonin treatment altered the expression of phospo-ERK1/2 in MDA-MB-231 cells, demonstrating that deltonin-induced apoptosis of MDA-MB-231 cells is associated with the inhibition of phosphorylation of AKT and ERK1/2.

In conclusion, our results demonstrate that the cytotoxic effect of deltonin in MDA-MB-231 cells is mediated by the induction of apoptosis. Deltonin-induced apoptosis involves the regulation of Bcl-2 family members and caspase-dependent mitochondrial dysfunction and also appears to be markedly associated with ROS production and regulation of ERK and AKT signaling pathways. Results indicate that deltonin may be an effective therapeutic agent for the treatment of breast cancer.

\section{Acknowledgements}

The present study was supported by grants from the the National Major Scientific and Technological Special Project (no. 2009ZX09102-045), National 12th 5-Year Plan Project of China (no. 2011BAJ07B04) and the National Natural Science Foundation of China (no. 20972105).

\section{References}

1. Jemal A, Bray F, Center MM, et al: Global cancer statistics. CA Cancer J Clin 61: 69-90, 2011.

2. Lambert R, Sauvaget $C$ and Sankaranarayanan R: Mass screening for colorectal cancer is not justified in most developing countries. Int J Cancer 125: 253-256, 2009.

3. Anderson BO, Shyyan R, Eniu A, et al: Breast cancer in limited-resource countries: An overview of the Breast Health Global Initiative 2005 Guidelines. Breast J 12 (Suppl 1): S3-S15, 2006.

4. Gottesman MM: Mechanisms of cancer drug resistance. Annu Rev Med 53: 615-627, 2002.

5. Efferth T, Li PC, Konkimalla VS and Kaina B: From traditional Chinese medicine to rational cancer therapy. Trends Mol Med 13: 353-361, 2007.
6. Chatterjee S, Kundu S and Bhattacharyya A: Mechanism of cadmium induced apoptosis in the immunocyte. Toxicol Lett 177: 83-89, 2008.

7. Pathak N and Khandelwal S: Role of oxidative stress and apoptosis in cadmium induced thymic atrophy and splenomegaly in mice. Toxicol Lett 169: 95-108, 2007.

8. Gupta S, Yel L, Kim D, et al: Arsenic trioxide induces apoptosis in peripheral blood T lymphocyte subsets by inducing oxidative stress: a role of Bcl-2. Mol Cancer Ther 2: 711-719, 2003.

9. Fan TJ, Han LH, Cong RS, et al: Caspase family proteases and apoptosis. Acta Biochim Biophys Sin (Shanghai) 37: 719-727, 2005.

10. Reed JC: Mechanisms of apoptosis. Am J Pathol 157: 1415-1430, 2000.

11. Ghobrial IM, Witzig TE and Adjei AA: Targeting apoptosis pathways in cancer therapy. CA Cancer J Clin 55: 178-194, 2005.

12. Desagher S and Martinou JC: Mitochondria as the central control point of apoptosis. Trends Cell Biol 10: 369-377, 2000.

13. Li H, Zhu H, Xu CJ and Yuan J: Cleavage of BID by caspase 8 mediates the mitochondrial damage in the Fas pathway of apoptosis. Cell 94: 491-501, 1998.

14. Green D and Kroemer G: The central executioners of apoptosis: caspases or mitochondria? Trends Cell Biol 8: 267-271, 1998.

15. Moalic S, Liagre B, Corbière C, et al: A plant steroid, diosgenin, induces apoptosis, cell cycle arrest and COX activity in osteosarcoma cells. FEBS Lett 506: 225-230, 2001.

16. Kaskiw MJ, Tassotto ML, Th'ng J and Jiang ZH: Synthesis and cytotoxic activity of diosgenyl saponin analogues. Bioorgan Med Chem 16: 3209-3217, 2008.

17. Kaskiw MJ, Tassotto ML, Mok M, et al: Structural analogues of diosgenyl saponins: synthesis and anticancer activity. Bioorgan Med Chem 17: 7670-7679, 2009.

18. Liu MJ, Yue PY, Wang Z and Wong RN: Methyl protodioscin induces $\mathrm{G} 2 / \mathrm{M}$ arrest and apoptosis in K562 cells with the hyperpolarization of mitochondria. Cancer Lett 224: 229-241, 2005.

19. Tong QY, Qing Y, Shu D, et al: Deltonin, a steroidal saponin, inhibits colon cancer cell growth in vitro and tumor growth in vivo via induction of apoptosis and antiangiogenesis. Cell Physiol Biochem 27: 233-242, 2011.

20. Shu D, Qing Y, Tong QY, et al: Deltonin isolated from Dioscorea zingiberensis inhibits cancer cell growth through inducing mitochondrial apoptosis and suppressing AKT and mitogen activated protein kinase signals. Biol Pharml Bull 34: 1231-1239, 2011.

21. Moon DO, Choi YH, Moon SK, et al: Gossypol decreases tumor necrosis factor- $\alpha$-induced intercellular adhesion molecule-1 expression via suppression of NF- $\kappa \mathrm{B}$ activity. Food Chem Toxicol 49: 999-1005, 2011.

22. Zhang Z, Li M, Wang H, et al: Antisense therapy targeting MDM2 oncogene in prostate cancer: effects on proliferation, apoptosis, multiple gene expression and chemotherapy. Proc Natl Acad Sci USA 100: 11636-11641, 2003.

23. Edwards LA, Thiessen B, Dragowska WH, et al: Inhibition of ILK in PTEN-mutant human glioblastomas inhibits PKB/Akt activation, induces apoptosis and delays tumor growth. Oncogene 24: 3596-3605, 2005.

24. Cossarizza A, Baccarani-Contri M, Kalashnikova G and Franceschi C: A new method for the cytofluorimetric analysis of mitochondrial membrane potential using the J-aggregate forming lipophilic cation 5,5',6,6'-tetrachloro-1,1',3,3'-tetraethylbenzimidazolcarbocyanine iodide (JC-1). Biochem Biophys Res Commun 197: 40-45, 1993.

25. Kroemer G, Zamzami N and Susin SA: Mitochondrial control of apoptosis. Immunol Today 18: 44-51, 1997.

26. Mitsopoulos P and Suntres ZE: Protective effects of liposomal N-Acetylcysteine against paraquat-induced cytotoxicity and gene expression. J Toxicol 2011: 1-14, 2011.

27. Konopleva M, Contractor R, Tsao T, et al: Mechanisms of apoptosis sensitivity and resistance to the BH3 mimetic ABT-737 in acute myeloid leukemia. Cancer Cell 10: 375-388, 2006.

28. Nguyen TT, Tran E, Nguyen TH, et al: The role of activated MEK-ERK pathway in quercetin-induced growth inhibition and apoptosis in A549 lung cancer cells. Carcinogenesis 25: 647-659, 2004.

29. Cusimano A, Foderà D, D'Alessandro N, et al: Potentiation of the antitumor effects of both selective cyclooxygenase- 1 and cyclooxygenase- 2 inhibitors in human hepatic cancer cells by inhibition of the MEK/ERK pathway. Cancer Biother 6: 1461-1468, 2007.

30. Kunnimalaiyaan M, Ndiaye M and Chen H: Apoptosis-mediated medullary thyroid cancer growth suppression by the PI3K inhibitor LY294002. Surgery 140: 1009-1014, 2006. 
31. Kong CS, Kimb JA, Yoon NY and Kim SK: Induction of apoptosis by phloroglucinol derivative from Ecklonia cava in MCF-7 human breast cancer cells. Food Chem Toxicol 47: 1653-1658, 2009.

32. Haruna S, Kuroi R, Kajiwara K, Hashimoto R, Matsugo S, Tokumaruc $S$ and Kojoa S: Induction of apoptosis in HL-60 cells by photochemically generated hydroxyl radicals. Bioorgan Med Chem Lett 12: 675-676, 2002.

33. Liu H, Xiao Y, Xiong C, Wei A and Ruan J: Apoptosis induced by a new flavonoid in human hepatoma HepG2 cells involves reactive oxygen species-mediated mitochondrial dysfunction and MAPK activation. Eur J Pharmacol 654: 209-216, 2011.

34. Kim MJ, Liao J, Dowling ML, et al: TRAIL inactivates the mitotic checkpoint and potentiates death induced by microtubule-targeting agents in human cancer cells. Cancer Res 68: 3440-3449, 2008.

35. Danial NN and Korsmeyer SJ: Cell death: critical control points Cell 116: 205-219, 2004.

36. Kluck RM, Bossy-Wetzel E, Green DR and Newmeyer DD: The release of cytochrome $\mathrm{c}$ from mitochondria: a primary site for Bcl-2 regulation of apoptosis. Science 275: 1132-1136, 1997.

37. Monteghirfo S, Tosetti F, Ambrosini C, et al: Antileukemia effects of xanthohumol in Bcr/Abl-transformed cells involve nuclear factor-kappaB and p53 modulation. Mol Cancer Ther 7: 2692-2702, 2008.

38. Won SJ, Chung KS, Ki YS, Choi JH, Cho WJ and Lee KT: CWJ-081, a novel 3-arylisoquinoline derivative, induces apoptosis in human leukemia HL-60 cells partially involves reactive oxygen species through c-Jun $\mathrm{NH} 2$-terminal kinase pathway. Bioorgan Med Chem Lett 20: 6447-6451, 2010.
39. Han YM, Shin DS, Lee YJ, Ismail IA, Hong SH, Han DC and Kwon BM: 2-Hydroxycurcuminoid induces apoptosis of human tumor cells through the reactive oxygen species-mitochondria pathway. Bioorgan Med Chem Lett 21: 747-751, 2011.

40. Dillon RL, White DE and Muller WJ: The phosphatidyl inositol 3-kinase signaling network: implications for human breast cancer. Oncogene 26: 1338-1345, 2007.

41. Viglietto G, Motti ML, Bruni P, et al: Cytoplasmic relocalization and inhibition of the cyclin-dependent kinase inhibitor p27 (Kip1) by PKB/Akt-mediated phosphorylation in breast cancer. Nat Med 8: 1136-1144, 2002.

42. Brunet A, Bonni A, Zigmond MJ, et al: AKT promotes cell survival by phosphorylating and inhibiting a Forkhead transcription factor. Cell 96: 857-868, 1999.

43. Bartkova J, Lukas J, Muller H, Lutzhoft D, Strauss M and Bartek J: Cyclin D1 protein expression and function in human breast cancer. Int J Cancer 57: 353-361, 1994.

44. Li Y, Corradetti MN, Inoki K and Guan KL: TSC2: filling the GAP in the mTOR signaling pathway. Trends Biochem Sci 29: 32-38, 2004.

45. Vivanco I and Sawyers CL: The phosphatidylinositol 3-kinase-AKT pathway in human cancer. Nat Rev Cancer 2: 489-501, 2002.

46. Fang JY and Richardson BC: The MAPK signaling pathways and colorectal cancer. Lancet Oncol 6: 322-327, 2005. 